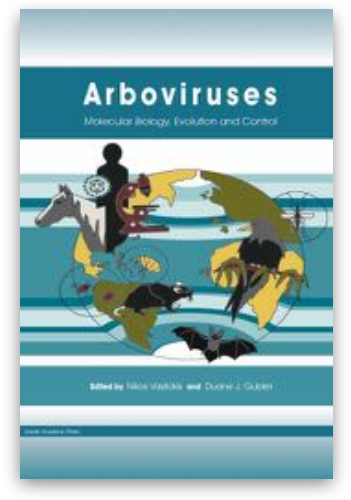

\section{Arboviruses Available Now!}

Molecular Biology, Evolution and Control Edited by: Nikos Vasilakis and Duane J. Gubler xii +398 pages, April 2016

Book: ISBN 978-1-910190-21-0, £159/ US\$319

Ebook: ISBN 978-1-910190-22-7, £159 / US\$319

This book brings together a panel of expert arbovirologists to produce a timely review of the rapidly expanding arbovirus research literature. In addition authors identify the most pressing questions that remain to be answered, thus providing a stimulus for future research. Topics include: taxonomy, genome organization, virus-host and virusvector interactions, evolutionary history, role of vertical transmission in arbovirus maintenance and evolution, epidemiology, arbovirus replication, pathogenesis, arbovirus diagnostics and control, including vaccines, novel antiviral drugs, RNA interference and genetically modified vectors. Essential reading for every arbovirologist and highly recommended for all virologists and public health officials.

Topics

- The Arboviruses: Quo Vadis?. - The Taxonomy of Arboviruses. • Genomic Organization of Arboviral Families. • Host Metabolism and its Contribution in Flavivirus Biogenesis. • Vector-borne Bunyavirus Pathogenesis and Innate Immune Evasion. • Vector-borne Rhabdoviruses. • Alphavirus - Host Interactions. - Molecular Interactions Between Arboviruses and Insect Vectors: Insects' Immune Responses to Virus Infection. • Genetic Diversity of Arboviruses. • Ecological and Epidemiological Factors Influencing Arbovirus Diversity, Evolution and Spread. • Role of Inter- and Intra-host Genetics in Arbovirus Evolution. • Arbovirus Genomics and Metagenomics. • Role of Vertical Transmission in Mosquito-borne Arbovirus Maintenance and Evolution. - The Boundaries of Arboviruses: Complexities Revealed in Their Host Ranges, Virus-Host Interactions and Evolutionary Relationships. - Laboratory Diagnosis of Arboviruses. • Conventional Vector Control: Evidence it Controls Arboviruses. • Biological Control of Arbovirus Vectors. $\bullet$ RNA Interference: A Pathway to Arbovirus Control. • Genetically Modified Vectors for Control of Arboviruses. • Arbovirus Vaccines. - Small Molecule Drug Development for Dengue Virus. • Arbovirology: Back to the Future.

Further details at: www.caister.com/arbo

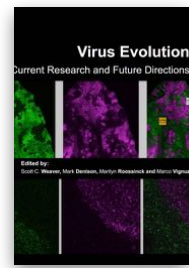

\title{
Virus Evolution
}

Current Research and Future Directions

Edited by: Scott C. Weaver, Mark Denison, Marilyn Roossinck and Marco Vignuzzi

366 pages, January 2016

Book: ISBN 978-1-910190-23-4, £159 / US\$319

Ebook: ISBN 978-1-910190-24-1, £159 / US\$319

- Viral Mutation Rates. $\bullet$ Viral Informatics: Tools for

Understanding the Evolution of biology's Most Varied Genomes.

- The Evolution and Transmission of Vector-borne Viruses. •

Choose Your Weapons: Origins and Evolution of Innate Host Defenses and Viral Counterstrategies. Evolution of the Interactions of Viruses with Their Plant Hosts. Evolution of Viral Virulence: Empirical Studies. - Taxonomy Advancement and Genome Size Change: Two Perspectives on RNA Virus Genetic Diversity. • Understanding Adaptation Through Experimental Evolution with Viruses: From Simple to Complex Environments. Evolution of Persistent Viruses in Plants. Paleovirology: The Study of Endogenous Viral Elements. • Population Genetic Modeling of Viruses. Emerging Viral Infections.

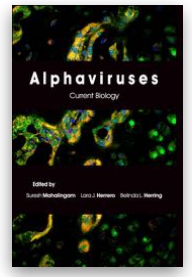
Alphaviruses
Current Biology
Edited by: Suresh Mahalingam, Lara Herrero and Belinda Herring
$x+184$ pages, January 2016
Book: ISBN 978-1-910190-15-9, £159 / US\$319
Ebook: ISBN 978-1-910190-16-6, £159 / US $\$ 319$

\section{Also of Interest}

- Molecular Virology and Control of Flaviviruses "a valuable resource" (Doodys)

- Bunyaviridae: Molecular and Cellular Biology Up-to-date reviews of current research in the field of Bunyaviridae molecular and cellular biology.

- Viruses and Interferon: Current Research

"a series of thorough reviews" (ISICR Newsletter)
- Alphavirus Genome Structure and Replication. • Alphavirus Evolution. - Laboratory Diagnosis and Detection of Alphaviruses. - The Interaction of Alphaviruses with the Interferon System. • Alphaviruses and their Role in Elucidating Antiviral Responses in Mosquitoes. • Animal Models of Alphavirus-induced Inflammatory Disease. $\bullet$ Clinical Manifestations of Arthritogenic Alphaviruses. Encephalitic Alphaviruses. • Application of Alphavirus Vectors for Gene Therapy. • Case Study: Chikungunya Virus. 


\section{Influenza}

Current Research

Edited by: Qinghua Wang and Yizhi Jane Tao

c. 192 pages, September 2016

Book: ISBN 978-1-910190-43-2, £159 / US\$319

Ebook: ISBN 978-1-910190-44-9, £159 / US $\$ 319$

- Stem-specific Broadly Neutralizing Antibodies of Influenza Virus Hemagglutinin. - Influenza Virus Replication and Transcription. • Recent Progress in Understanding Influenza B Virus Hemagglutinin. • Structure and Assembly of the Influenza A Virus Ribonucleoprotein Complex. • Host Factors Regulating the Influenza Virus Replication Machinery. $\bullet$ Receptor Specificity in Surveillance of Natural Sequence Evolution of Influenza A Virus Hemagglutinin. - PB1-F2: A Multifunctional Non-structural Influenza A Virus Protein. • Avian Influenza H7N9 Virus.
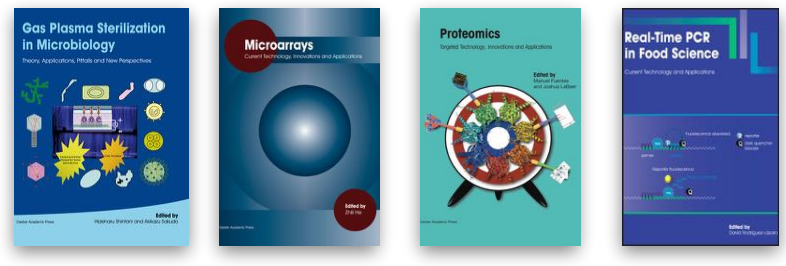

Advanced Vaccine Research Methods for the Decade of Vaccines

Edited by: F Bagnoli, R Rappuoli

A thorough and up-to-date review of vaccinology research in age of omics technologies.

Antibiotics: Current Innovations and Future Trends

Edited by: S Sánchez, AL Demain

"genuinely a brilliant resource" (ChemMedChem)

Applied RNAi: From Fundamental Research to Therapeutic Applications

Edited by: P Arbuthnot, MS Weinberg

"an excellent resource" (Doodys); "I would recommend this book" (ChemMedChem)

\section{Cytomegaloviruses: From Molecular Pathogenesis to} Intervention

Edited by: MJ Reddehase

"intended for a wide audience" Ref. Res. Book News

Rhabdoviruses: Molecular Taxonomy, Evolution, Genomics, Ecology, Host-Vector Interactions, Cytopathology and Control Edited by: RG Dietzgen, IV Kuzmin

"goes way beyond what can be found in other textbooks" (ASM Microbe)

\section{Small DNA Tumour Viruses}

Edited by: K Gaston

"invaluable for its multiple perspectives and concise summary of a large body of research" (MedicalScienceBooks.com)

Hepatitis C: Antiviral Drug Discovery and Development Edited by: SL Tan, Y He

"in-depth information" (Microbiol. Today)

Frontiers in Dengue Virus Research

Edited by: KA Hanley and SC Weaver

"a valuable point of reference" (Microbiol. Today)

\section{Books of Related interest}

\section{MALDI-TOF Mass Spectrometry in Microbiology}

Edited by: Markus Kostrzewa and Sören Schubert

C. 200 pages, July 2016

Book: ISBN 978-1-910190-41-8, £159 / US\$319

Ebook: ISBN 978-1-910190-42-5, £159 / US\$319

-A Personal Vision of the MALDI-TOF-MS Journey from Obscurity to Frontline Diagnostics. • Matrix Assisted Laser Desorption Ionization Time-of-Flight Mass Spectrometry for the Clinical Laboratory. • Analysis of Anaerobes and Some Other Fastidious Bacteria. • Identification, Typing and Susceptibility Testing of Fungi (incl. Yeasts) by MALDI-TOF MS. - Molecular Typing of Bacteria/Fungi Using MALDI-TOF MS. • Matrix-assisted Laser Desorption/lonization Timeof-flight Mass Spectrometry for Determination of Resistance to Antibiotics. - Application of MALDI-TOF MS in Veterinary and Food Microbiology. • MALDI-TOF MS for Environmental Analysis,

Microbiome Research and as a Tool for Biological Resource Centres. - The World of Nucleic Acid Based Mass Spectrometry for Microbial and Viral Detection. - Future Trends and Perspectives of MALDI-TOF Mass Spectrometry in the Microbiology Laboratory.

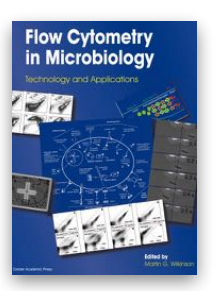

Flow Cytometry in Microbiology

Technology and Applications

Edited by: Martin G. Wilkinson xii +218 pages, September 2015 Book: ISBN 978-1-910190-11-1, £159 / US\$319 Ebook: ISBN 978-1-910190-12-8, £159 / US\$319

"an impressive group of experts" (ProtoView)

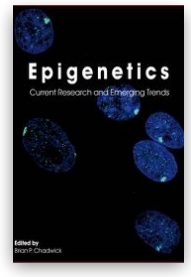

\section{Epigenetics}

Current Research and Emerging Trends Edited by: Brian P. Chadwick

xii +354 pages, July 2015

Book: ISBN 978-1-910190-07-4, £159 / US\$319

Ebook: ISBN 978-1-910190-08-1, £159 / US $\$ 319$

"this is one text you don't want to miss" (Epigenie), "up-to-date information" (ChemMedChem)

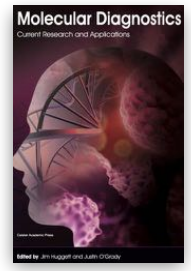

\section{Molecular Diagnostics}

Current Research and Applications

Edited by: Jim Huggett and Justin O'Grady xii +248 pages, May 2014

Book: ISBN 978-1-908230-41-6, £159 / US\$319

Ebook: ISBN 978-1-908230-64-5, £159 / US\$319

- Molecular Diagnostics: An Introduction. • Transcriptome-based Biomarkers: A Road Map Exemplified for Peripheral Blood-based Biomarker Discovery, Development and Clinical Use. • Development of Methylation Biomarkers for Clinical Applications and Methylation-sensitive Highresolution Melting (MS-HRM) Technology. • Genetic and Epigenetic Biomarkers of Colorectal Cancer. $\bullet$ Molecular Diagnosis in Medical Microbiology: The Horizon Draws Near. • Molecular Diagnostics: Current Research and Applications. X XMRV: A Cautionary Tale. Ancient DNA and the Fingerprints of Disease: Retrieving Human Pathogen Genomic Sequences from Archaeological Remains Using Real-time Quantitative Polymerase Chain Reaction. • Point-of-Care Nucleic Acid Testing: User Requirements, Regulatory Affairs, and Quality Assurance. • Point-of-Care Nucleic Acid Testing: Clinical Applications and Current Technologies. - From Bench to Bedside: Development of Polymerase Chain Reaction Integrated Systems in the Regulated Markets. • Future of Molecular Diagnostics: The Example of Infectious Diseases.

"I would highly recommend this book" (Doodys); "useful new insights" (Aus. J. Med. Sci.) 